\title{
Maternal and paternal family history of type 2 diabetes differently influence lipid parameters in young nondiabetic Japanese women
}

\author{
Kemal Sasaki - Aya Yoshida $\cdot$ Hiroshi Ohta Yoshiharu Aizawa \\ Akiko Kojima · Hitomi Chiba $\cdot$ Shin Mizuguchi $\cdot$ Tatsunori Ishidzuka • \\ Hiroshi Goto $\cdot$ Chiho Uegaki $\cdot$ Kyuhei Kotake
}

Received: 1 April 2012/ Accepted: 5 July 2012/Published online: 25 July 2012

(C) The Japanese Society for Hygiene 2012

\begin{abstract}
Objectives We assessed the association of family history of type 2 diabetes (T2D) with parameters used for health checkups in young Japanese women.

Methods The subjects were 497 nondiabetic women aged 19-39 years. Among them, the mothers of 34 subjects and fathers of 50 had T2D (MD group and PD group, respectively). The subjects were assessed for levels of total cholesterol (TC), low-density lipoprotein cholesterol (LDL-C), high-density lipoprotein cholesterol (HDL-C), and triglyceride (TG).

Results TC and LDL-C level showed a tendency to increase in the MD group compared with subjects without family history of T2D. LDL-C/HDL-C ratio $\geq 2.14$ was found in 32.4 and $18.0 \%$ of subjects in the MD and $\mathrm{PD}$ groups, respectively. When adjusted for differences in age, body mass index, smoking status, and drinking habits, the
\end{abstract}

K. Sasaki - A. Yoshida - A. Kojima - H. Chiba ·

S. Mizuguchi · T. Ishidzuka · H. Goto · C. Uegaki - K. Kotake

Sagamihara Public Health Center, 6-1-1 Fujimi, Chuo-ku,

Sagamihara, Kanagawa 252-5277, Japan

Present Address:

K. Sasaki $(\square)$

Aichi Children's Health and Medical Center,

1-2 Osakada, Morioka-cho, Obu, Aichi 474-8710, Japan

e-mail: kemal-s@umin.ac.jp

H. Ohta

Department of Public Health, Kitasato University School of Medicine, 1-15-1 Kitasato, Minami-ku, Sagamihara,

Kanagawa 252-0374, Japan

\section{Y. Aizawa}

Department of Preventive Medicine, Kitasato University School of Medicine, 1-15-1 Kitasato, Minami-ku, Sagamihara,

Kanagawa 252-0374, Japan
MD group was found to have a higher risk of abnormal TC and LDL-C levels than the PD group. LDL-C/HDL-C ratio was independently associated with maternal family history but not with paternal family history (odds ratio 3.44 [99 \% confidence interval 1.11-10.6] and 1.21 [0.38-3.89], respectively). There was no association between TG/HDL$\mathrm{C}$ ratio and family history type of T2D.

Conclusions Maternal family history of T2D had a more pronounced effect on the lipid parameters generally evaluated during health checkups than did paternal family history of T2D. Therefore, we recommend systematic screening for early detection and appropriate healthcare guidance for Japanese women, particularly those with maternal family history of T2D.

Keywords Diabetes - Family history - Dyslipidemia . Low-density lipoprotein cholesterol/high-density lipoprotein cholesterol ratio $\cdot$ Health checkup

\section{Introduction}

Genetic factors and lifestyle play important roles in the risk of type 2 diabetes (T2D) [1]. Recent genetic studies have revealed the association of several genomic loci with the risk of T2D [2]. However, the application of genetic testing in general medical practice has legal and ethical issues. Family history of T2D is the simplest and most easily available genetic information [3]. Family history of T2D increases the risk of insulin resistance and accelerates the onset of T2D in the offspring $[4,5]$. Moreover, family history of T2D is related with worse atherosclerotic risk factors, including decreased high-density lipoprotein cholesterol (HDL-C) levels [6] and increased body mass index (BMI) [5-7], and plasma concentrations of hepatic 
enzymes [8], total cholesterol (TC) [7], low-density lipoprotein cholesterol (LDL-C) [4], and triglyceride (TG) [6, 7]. These findings show an association between a genetic predisposition to T2D and risk of atherosclerotic disease.

Several studies have shown a predominance of maternal family history of T2D in patients [4, 9-11]. The proposed explanations for excess maternal transmission of T2D include mitochondrial DNA mutations [12], intrauterine environment [13], and behavioral influence of the mother $[14,15]$. Some studies have compared maternal and paternal family history for insulin resistance, but limited and controversial data are available on the relationship between the type of family history and other atherosclerotic risk factors, including dyslipidemia $[4,6,10,11,16]$.

Most atherosclerotic risk factors, other than being overweight, cause asymptomatic changes until the development of severe complications. Health checkups provide a valuable chance for early detection of such asymptomatic abnormalities. Therefore, it is valuable to identify how the type of family history of T2D influences atherosclerotic risk factors measured during health checkups. In this study, we examined whether maternal and paternal family history of T2D are associated with the clinical parameters commonly evaluated during health checkups in young nondiabetic Japanese women.

\section{Materials and methods}

\section{Study population}

The subjects were residents of Sagamihara City, Kanagawa Prefecture, Japan. The city is a typical modern Japanese city with population of 700,000 , and $29 \%$ of individuals are between 19 and 39 years of age. The data were collected during health checkups for individuals between 19 and 39 years of age, conducted by the public health center from April 2008 to March 2010. The health checkups were performed for 189 male and 685 women, and this study was carried out with women. A total of 497 women were analyzed in this study. Inclusion criteria included women, and age between 19 and 39 years. Exclusion criteria included non-Japanese ( 8 women), multiple visit of health checkup (172 women), combination of maternal and paternal family history of T2D (8 women), pregnant (none), and treatment for ischemic heart disease, hypertension, liver dysfunction, dyslipidemia, or diabetes (none). Physicians asked each subject about their smoking and drinking habits as well as family history. The subjects were divided into 3 groups: (1) 413 women with no family history of T2D (ND), defined as having neither parents nor second-degree relatives with T2D, (2) 34 women with maternal family history of T2D (MD), defined as having mothers with T2D, and (3) 50 women with paternal family history of T2D (PD), defined as having fathers with T2D. The numbers of subject who had family history of dyslipidemia were as follows: ND group, 32 (7.7\%); MD group, $4(11.8 \%)$; and PD group, $4(8.0 \%)$.

The data were anonymized without any links to personal data. This study was performed in accordance with the recommendations outlined in the Declaration of Helsinki and with the ethics guidelines for epidemiological research laid down by the Ministry of Education, Culture, Sports, Science, and Technology and by the Ministry of Health, Labor, and Welfare. Data evaluation was ethically approved by Sagamihara Public Health Center Review Board.

\section{Analysis}

After an overnight fast, subjects underwent a health checkup at the health center between 0900 and 1100 hours. The height and weight of each subject were measured with the subject wearing indoor clothes but no shoes. Blood pressure (BP) was measured by a physician with a mercury sphygmomanometer on the right arm of seated subjects after a 5-min rest. Systolic blood pressure (SBP) was recorded at the appearance of sounds, and diastolic blood pressure (DBP) was recorded at the disappearance of sounds (V-phase Korotkov). Blood samples were obtained to perform measurements of aspartate aminotransferase (AST), alanine aminotransferase (ALT), gamma-glutamyl transpeptidase (GGT), TC, HDL-C, TG, glucose (fasting blood sugar, FBS), and hemoglobin Alc (HbAlc). Biochemical measurements were performed at the Health Sciences Research Institute, Inc. (Yokohama, Japan) by using routine laboratory methods. LDL-C was calculated as TC - HDL-C - TG $\times 0.2$, according to the Friedewald formula [17]. There were no subjects with TG level $\geq 400 \mathrm{mg} / \mathrm{dL}$. The cutoff values for abnormality were as follows: obesity, BMI $\geq 25 \mathrm{~kg} / \mathrm{m}^{2}$; high blood pressure, SBP $\geq 130 \mathrm{mmHg}$ and/or DBP $\geq 85 \mathrm{mmHg}$; AST level $>40$ IU/L; ALT level $>45$ IU/L; GGT level $>32$ IU/L; TC level $\geq 220 \mathrm{mg} / \mathrm{dL}$, LDL-C level $\geq 140 \mathrm{mg} / \mathrm{dL}$; HDL-C level $<40 \mathrm{mg} / \mathrm{dL}$; TG level $\geq 150 \mathrm{mg} / \mathrm{dL}$; LDL-C/HDL-C ratio $\geq 2.14$; TG/HDL-C ratio $\geq 1.5$; FBS level $\geq 100 \mathrm{mg} /$ $\mathrm{dL}$; and $\mathrm{HbA} 1 \mathrm{c}$ level $\geq 5.3 \%$. The criteria for high blood pressure and hyperglycemia were set at the high end of the normal range defined by the Japanese Society of Hypertension [18] and Japan Diabetes Society [19]. The criteria for ratios of lipid components were based on a recently reported threshold for identifying insulin resistance in nonobese Japanese people [20]. The criterion for HbA1c was set as the value corresponding to the cutoff point for the high end of the normal range of FBS in Japanese people [21]. 
Table 1 Mean \pm standard deviation (SD) for each item in subjects stratified by family history of type 2 diabetes

\begin{tabular}{|c|c|c|c|c|}
\hline & $\mathrm{ND}^{\mathrm{a}}$ & MD & PD & $P$ value ${ }^{\S}$ \\
\hline Age (years) & $33.4 \pm 4.1$ & $33.7 \pm 3.2$ & $34.1 \pm 3.2$ & - \\
\hline BMI $\left(\mathrm{kg} / \mathrm{m}^{2}\right)$ & $21.0 \pm 3.0$ & $21.5 \pm 3.3$ & $22.1 \pm 3.5$ & - \\
\hline $\mathrm{SBP}(\mathrm{mmHg})$ & $105 \pm 12$ & $104 \pm 12$ & $106 \pm 12$ & 0.649 \\
\hline $\mathrm{DBP}(\mathrm{mmHg})$ & $67 \pm 9$ & $70 \pm 10$ & $67 \pm 10$ & 0.259 \\
\hline AST (IU/L) & $18 \pm 4$ & $17 \pm 3$ & $18 \pm 5$ & 0.282 \\
\hline ALT (IU/L) & $15 \pm 8$ & $15 \pm 5$ & $15 \pm 9$ & 0.661 \\
\hline GGT (IU/L) & $19 \pm 16$ & $21 \pm 18$ & $22 \pm 23$ & 0.456 \\
\hline $\mathrm{TC}(\mathrm{mg} / \mathrm{dL})$ & $187 \pm 31$ & $202 \pm 35$ & $198 \pm 35$ & 0.018 \\
\hline LDL-C (mg/dL) & $103 \pm 27$ & $117 \pm 29$ & $109 \pm 28$ & 0.042 \\
\hline HDL-C (mg/dL) & $71 \pm 15$ & $71 \pm 18$ & $74 \pm 17$ & 0.097 \\
\hline $\mathrm{TG}(\mathrm{mg} / \mathrm{dL})$ & $67 \pm 31$ & $71 \pm 35$ & $75 \pm 50$ & 0.666 \\
\hline LDL-C/HDL-C & $1.53 \pm 0.52$ & $1.75 \pm 0.65$ & $1.56 \pm 0.57$ & 0.115 \\
\hline TG/HDL-C & $1.01 \pm 0.61$ & $1.11 \pm 0.75$ & $1.16 \pm 1.15$ & 0.833 \\
\hline FBS $(\mathrm{mg} / \mathrm{dL})$ & $85 \pm 7$ & $86 \pm 6$ & $86 \pm 7$ & 0.712 \\
\hline HbA1c (\%) & $4.8 \pm 0.2$ & $4.9 \pm 0.3$ & $4.8 \pm 0.2$ & 0.197 \\
\hline
\end{tabular}

$N D$ subjects with no family history of type 2 diabetes, $M D$ subjects with maternal family history of type 2 diabetes, $P D$ subjects with paternal family history of type 2 diabetes, $B M I$ body mass index, SBP systolic blood pressure, $D B P$ diastolic blood pressure, AST aspartate aminotransferase, $A L T$ alanine aminotransferase, $G G T$ gamma-glutamyl transpeptidase, $T C$ total cholesterol, $L D L-C$ low-density lipoprotein-cholesterol, $H D L-C$ high-density lipoprotein-cholesterol, $T G$ triglyceride, $F B S$ glucose, $H b A l c$ hemoglobin A1c

$\S$ Adjusted for age, BMI, smoking status (pack-years), and drinking status (ethanol consumption per day)

${ }^{a}$ Values are mean $\pm \mathrm{SD}$

\section{Statistical analysis}

Analysis of covariance was used to assess the significance of differences as mean values. Multiple logistic regression analysis was used to examine the significance of odds ratio (OR) for abnormality as compared with the ND group. The covariates for both methods are as follows: age, BMI, smoking status (pack-years), and drinking habits (ethanol consumption per day). The Pearson correlation coefficient was used to assess the correlation between FBS or HbA1c levels and other components. Analyses were performed using SPSS statistics for Macintosh version 18. $P$ value $<0.01$ was considered significant.

\section{Results}

Table 1 presents the mean values of measured parameters stratified by family history of T2D. TC and LDL-C levels showed a tendency to vary among the family history type of T2D: TC and LDL-C levels were the highest in the MD group among the three groups. The estimated mean values of TC and LDL-C levels in the MD group were also the highest among all groups. The estimated mean \pm standard error for TC and LDL-C (mg/dL) were as follows: for TC: ND group, $187 \pm 2$; MD group, $201 \pm 6$; and PD group, $197 \pm 5$; and for LDL-C: ND group, $104 \pm 1$; MD group, $115 \pm 5$; and PD group, $107 \pm 4$.
The overall prevalence of obesity was $12 \%$, and there were no significant differences in the prevalence of obesity among all groups (Fisher's exact test; $P=$ 1.000). Increase of TC levels and LDL-C/HDL-C ratios were the most common abnormalities in the MD group, which reached over $30 \%$ (Table 2). Abnormal TC level was also the most common abnormality in the PD group. Both family history types of T2D showed a tendency to be a risk factor for abnormal GGT level. Maternal family history tended to be a risk factor for abnormal LDL-C level. Paternal family history was a significant risk factor for abnormal TC level under the unadjusted condition (OR 2.53 [99\% confidence interval 1.04-6.18]). However, only when BMI was added as a covariate in logistic regression analysis, paternal family history was no longer independently associated with TC (OR 2.26 [0.87-5.84]).

In the MD group, $\mathrm{HbA1c}$ level tended to correlate with HDL-C levels, and LDL-C/HDL-C and TG/HDL-C ratios (Table 3). In the PD group, FBS level was correlated not only with lipid parameters, TG levels, LDL-C/HDL-C, and TG/HDL-C ratios but also with BMI.

\section{Discussion}

In this study, we investigated the effects of family history of T2D on clinical parameters commonly evaluated during 
Table 2 Abnormal values in subjects stratified by family history of type 2 diabetes

\begin{tabular}{|c|c|c|c|c|c|}
\hline & \multirow[t]{2}{*}{$\mathrm{ND}^{\mathrm{a}}$} & \multirow[t]{2}{*}{ MD } & \multirow[t]{2}{*}{ PD } & \multicolumn{2}{|l|}{ Odds ratio $^{\mathrm{b}}$} \\
\hline & & & & MD vs. ND & PD vs. ND \\
\hline Obesity & 47 (11.4) & $3(8.8)$ & $5(10.0)$ & - & - \\
\hline High blood pressure & $16(3.9)$ & $2(5.9)$ & $1(2.0)$ & $1.53[0.21-11.8]^{\mathrm{c}}$ & $0.41[0.03-6.63]$ \\
\hline AST & $1(0.2)$ & $0(0)$ & $0(0)$ & - & - \\
\hline ALT & $5(1.2)$ & $0(0)$ & $1(2.0)$ & - & $0.80[0.03-21.8]$ \\
\hline GGT & $24(5.81)$ & $6(17.6)$ & $8(16.0)$ & $3.76[0.98-14.5]$ & $3.03[0.92-10.1]$ \\
\hline $\mathrm{TC}$ & $55(13.3)$ & $11(32.4)$ & $14(28.0)$ & 3.04 [1.02-8.99] & $2.37[0.90-6.17]$ \\
\hline LDL-C & $39(9.4)$ & $8(23.5)$ & $10(20.0)$ & $3.17[0.92-10.9]$ & $2.16[0.70-6.45]$ \\
\hline HDL-C & $1(0.2)$ & $0(0)$ & $0(0)$ & - & - \\
\hline TG & $12(2.9)$ & $2(5.9)$ & $2(4.0)$ & $2.15[0.25-18.6]$ & $0.86[0.09-8.74]$ \\
\hline LDL-C/HDL-C & $53(12.8)$ & $11(32.4)$ & $9(18.0)$ & $3.44[1.11-10.6]$ & $1.21[0.38-3.89]$ \\
\hline TG/HDL-C & $55(13.3)$ & $4(11.8)$ & $9(18.0)$ & $0.76[0.17-3.42]$ & $1.15[0.37-3.51]$ \\
\hline FBS & $9(2.2)$ & $1(2.9)$ & $2(4.0)$ & $1.04[0.07-12.9]$ & $0.92[0.07-12.0]$ \\
\hline $\mathrm{HbA1c}$ & $8(1.9)$ & $3(10.0)$ & $1(2.0)$ & $5.30[0.83-34.1]$ & $1.01[0.06-16.5]$ \\
\hline
\end{tabular}

$N D$ subjects with no family history of type 2 diabetes, $M D$ subjects with maternal family history of type 2 diabetes, $P D$ subjects with paternal family history of type 2 diabetes, $A S T$ aspartate aminotransferase, $A L T$ alanine aminotransferase, $G G T$ gamma-glutamyl transpeptidase, $T C$ total cholesterol, $L D L-C$ low-density lipoprotein-cholesterol, $H D L-C$ high-density lipoprotein-cholesterol, $T G$ triglyceride, $F B S$ glucose, $H b A l c$ hemoglobin A1c

${ }^{a}$ Values in parentheses are percentage of subjects in each group. The thresholds for each abnormality are described in the "Materials and methods" section

b Adjusted for age, BMI, smoking status (pack-years), and drinking status (ethanol consumption per day)

c $99 \%$ confidence interval

Table 3 Pearson's correlation coefficient in subjects stratified by family history of type 2 diabetes

\begin{tabular}{|c|c|c|c|c|c|c|c|c|}
\hline & \multicolumn{4}{|l|}{ MD } & \multicolumn{4}{|l|}{ PD } \\
\hline & \multicolumn{2}{|l|}{ FBS } & \multicolumn{2}{|l|}{ HbA1c } & \multicolumn{2}{|l|}{ FBS } & \multicolumn{2}{|l|}{ HbA1c } \\
\hline & $r$ & $P$ & $r$ & $P$ & $r$ & $P$ & $r$ & $P$ \\
\hline Age & 0.214 & 0.256 & 0.233 & 0.215 & -0.238 & 0.111 & 0.124 & 0.410 \\
\hline BMI & 0.220 & 0.243 & 0.183 & 0.332 & 0.442 & 0.002 & -0.030 & 0.841 \\
\hline SBP & 0.091 & 0.631 & 0.089 & 0.640 & 0.335 & 0.023 & 0.037 & 0.808 \\
\hline DBP & 0.153 & 0.418 & 0.160 & 0.398 & 0.102 & 0.501 & 0.090 & 0.550 \\
\hline AST & -0.241 & 0.200 & -0.199 & 0.292 & 0.047 & 0.756 & -0.041 & 0.785 \\
\hline ALT & 0.061 & 0.749 & -0.059 & 0.758 & 0.246 & 0.100 & 0.060 & 0.690 \\
\hline GGT & 0.182 & 0.335 & -0.253 & 0.178 & 0.211 & 0.159 & 0.032 & 0.832 \\
\hline $\mathrm{TC}$ & 0.079 & 0.676 & 0.036 & 0.849 & 0.146 & 0.333 & -0.026 & 0.863 \\
\hline LDL-C & 0.063 & 0.743 & 0.206 & 0.275 & 0.225 & 0.132 & 0.019 & 0.902 \\
\hline HDL-C & 0.038 & 0.843 & -0.399 & 0.029 & -0.320 & 0.030 & -0.064 & 0.671 \\
\hline TG & 0.037 & 0.847 & 0.317 & 0.087 & 0.441 & 0.002 & -0.035 & 0.818 \\
\hline LDL-C/HDL-C & 0.023 & 0.904 & 0.389 & 0.033 & 0.455 & 0.001 & 0.052 & 0.731 \\
\hline TG/HDL-C & 0.018 & 0.925 & 0.385 & 0.036 & 0.437 & 0.002 & -0.057 & 0.706 \\
\hline
\end{tabular}

$M D$ subjects with maternal family history of type 2 diabetes, $P D$ subjects with paternal family history of type 2 diabetes, $B M I$ body mass index, $S B P$ systolic blood pressure, $D B P$ diastolic blood pressure, $A S T$ aspartate aminotransferase, $A L T$ alanine aminotransferase, $G G T$ gammaglutamyl transpeptidase, $T C$ total cholesterol, $L D L-C$ low-density lipoprotein-cholesterol, $H D L-C$ high-density lipoprotein-cholesterol, $T G$ triglyceride, FBS glucose, $H b A 1 c$ hemoglobin A1c

health checkups in young nondiabetic Japanese women. Family history was associated with variations in the levels of lipid parameters, such as TC level and the LDL-C/HDL-
$\mathrm{C}$ ratio. On the whole, maternal family history had a more potent influence on these factors than did paternal family history. Both HbA1c levels in subjects with maternal 
family history and FBS levels in those with paternal family history tended to correlate with lipid parameters.

In this study, maternal family history showed more impact on lipid abnormalities, probably because of disparities between family history types in terms of insulin resistance. Several studies have demonstrated more potent insulin resistance in subjects with maternal family history than in those with paternal family history $[6,16,22]$. The effect of insulin resistance on the metabolism of very lowdensity lipoprotein and HDL-C is greater than its effect on LDL-C metabolism [23, 24], and increased TG/HDL-C ratio is a good marker of insulin resistance [25]. Notably, those with maternal family history of T2D had a higher association with the LDL-C/HDL-C ratio than with the TG/ HDL-C ratio in the current study. This result is consistent with a recent study among nondiabetic Japanese subjects, where the LDL-C/HDL-C ratio was a better marker for insulin resistance than was the TG/HDL-C ratio [20]. Our study shared similar conditions with their study; a large majority of our subjects were nonobese Japanese women, and their subjects were also nonobese Japanese. In Japanese subjects, the LDL-C/HDL-C ratio is a better predictor of atherosclerotic disease, and LDL-C is more closely related with metabolic syndrome in women than in men $[26,27]$. Taken together, these results suggest that Japanese women with maternal family history of T2D need appropriate preventive measures against atherosclerotic disease.

The contribution of family history type of T2D on lipids has been controversial in both diabetics and nondiabetics $[4,6,10,11,16]$. The results of this study show a greater impact of maternal family history on lipid parameters than paternal family history, which is in accord with a previous study of nondiabetic South East Asians, composed of $68 \%$ Chinese, $18 \%$ Malays, and $14 \%$ Asian Indians [6]. However, there were some contradictions between the previous and current study: in the previous study, not observed in current study, maternal family history was associated with lower HDL-C level and higher TG level [6]. In a multicenter European study, although there was no reference to ethnic composition, no difference was observed in LDL-C, HDL-C, and TG levels between subjects with maternal and paternal family history [16]. The different contributions of family history in previous studies and the current study could be partly due to ethnic variations, including lifestyle. This hypothesis is supported by the finding that the dietary habits among Asians, including Japanese, influenced fasting serum insulin and lipid levels [28].

Previous reports have shown that lipid parameters are more highly correlated with HbA1c than with FBS [29, 30]. Consistent with previous studies, lipid parameters tended to correlate with $\mathrm{HbA} 1 \mathrm{c}$ rather than FBS in the MD group. However, the lipid parameters in the PD group were better correlated with FBS than HbA1c. The logistic regression analysis revealed that BMI interfered with the association between TC and paternal family history. BMI was correlated with FBS in the PD group. These results suggest an interaction between BMI and the correlation between glucose and lipid metabolism parameters in subjects with paternal family history of T2D.

There are some limitations of this study. The first limitation is the small sample size, which implies the need to take care not to overinterpret our results. Second, this study did not measure insulin resistance; therefore, it could not assess a direct association with family history of T2D. Rather, this study was designed to assess the contribution of family history to data from clinical items commonly used in health checkups. Third, this study lacks data on relevant phenotypic characteristics (e.g., age at diagnosis, antidiabetic treatment, or complications) of T2D in the parents. Based on these limitations, further studies are required to elucidate the mechanism that underlies these associations of family history of T2D with lipid parameters. However, the current study shows for the first time that the two types of family history of T2D differently influenced such parameters in young Japanese women, providing clues to the relationship between the type of family history and atherosclerotic risk.

In conclusion, we found a more pronounced potential of maternal family history of T2D for exacerbation of lipid parameters generally evaluated during health checkups than paternal family history of T2D, in young Japanese women. The subjects with maternal family history of T2D had higher risk of elevated LDL-C/HDL-C ratio, a pivotal atherosclerotic index. As these anomalies are asymptomatic at an early stage, systematic screening and appropriate healthcare guidance are required for subjects with maternal family history of T2D.

Conflict of interest The authors state that they have no conflict of interest (COI).

\section{References}

1. O'Rahilly S. Non-insulin dependent diabetes mellitus: the gathering storm. BMJ. 1997;314:955-9.

2. Okamoto K, Iwasaki N, Nishimura C, Doi K, Noiri E, Nakamura $\mathrm{S}$, et al. Identification of $\mathrm{KCNJ} 15$ as a susceptibility gene in Asian patients with type 2 diabetes mellitus. Am J Hum Genet. 2010;86:54-64.

3. Harrison TA, Hindorff LA, Kim H, Wines RC, Bowen DJ, McGrath BB, et al. Family history of diabetes as a potential public health tool. Am J Prev Med. 2003;24:152-9.

4. Bo S, Cavallo-Perin P, Gentile L, Repetti E, Pagano G. Influence of a familial history of diabetes on the clinical characteristics of patients with type 2 diabetes mellitus. Diabet Med. 2000;17:538-42.

5. Vaag A, Lehtovirta M, Thye-Rönn P, Groop L. European Group of Insulin Resistance. Metabolic impact of a family history of Type 2 diabetes. Results from a European multicentre study (EGIR). Diabet Med. 2001;18:533-40. 
6. Tan JT, Tan LS, Chia KS, Chew SK, Tai ES. A family history of type 2 diabetes is associated with glucose intolerance and obesity-related traits with evidence of excess maternal transmission for obesity-related traits in a South East Asian population. Diabetes Res Clin Pract. 2008;82:268-75.

7. van der Sande MA, Walraven GE, Milligan PJ, Banya WA, Ceesay SM, Nyan OA, et al. Family history: an opportunity for early interventions and improved control of hypertension, obesity and diabetes. Bull World Health Organ. 2001;79:321-8.

8. Inoue K, Matsumoto M, Miyoshi Y, Kobayashi Y. Elevated liver enzymes in women with a family history of diabetes. Diabetes Res Clin Pract. 2008;79:e4-7.

9. Karter AJ, Ferrara A, Rowell SE, Selby JV, Ackerson LM, Newman B, et al. Excess maternal transmission of type 2 diabetes. Diabet Care. 1999;22:938-43.

10. Scheffel RS, Kramer CK, Rados DV, Pinto LC, Crispim D, Gross $\mathrm{JL}$, et al. The prevalence of chronic diabetic complications and metabolic syndrome is not associated with maternal type 2 diabetes. Braz J Med Biol Res. 2008;41:1123-8.

11. Papazafiropoulou A, Sotiropoulos A, Skliros E, Kardara M, Kokolaki A, Apostolou O, et al. Familial history of diabetes and clinical characteristics in Greek subjects with type 2 diabetes. BMC Endocr Disord. 2009;12:9.

12. Rotig A, Bonnefont JP, Munnich A. Mitochondrial diabetes mellitus. Diabetes Metab. 1996;22:291-8.

13. Pettitt DJ, Aleck KA, Baird HR, Carraher MJ, Bennett PH, Knowler WC. Congenital susceptibility to NIDDM: role of intrauterine environment. Diabetes. 1988;37:622-8.

14. Hales CN, Barker DJ. Type 2 (non-insulin-dependent) diabetes mellitus: the thrifty phenotype hypothesis. Diabetologia. 1992;35: 595-601.

15. Mayer EJ, Newman B, Austin MA, Zhang D, Quesenberry CP Jr, Edwards $\mathrm{K}$, et al. Genetic and environmental influences on insulin levels and the insulin resistance syndrome: an analysis of women twins. Am J Epidemiol. 1996;143:323-32.

16. Natali A, Muscelli E, Mari A, Balkau B, Walker M, Tura A, et al. Insulin sensitivity and beta-cell function in the offspring of type 2 diabetic patients: impact of line of inheritance. J Clin Endocrinol Metab. 2010;95:4703-11.

17. Friedewald WT, Levy RI, Fredrickson DS. Estimation of the concentration of low-density lipoprotein cholesterol in plasma, without use of the preparative ultracentrifuge. Clin Chem. 1972;18:499-502.

18. Ogihara T, Kikuchi K, Matsuoka H, Fujita T, Higaki J, Horiuchi $\mathrm{M}$, et al. The Japanese Society of Hypertension guidelines for the management of hypertension (JSH 2009). Hypertens Res. 2009; $32: 3-107$.
19. Kadowaki T, Haneda M, Tominaga M, Yamada N, Iwamoto Y, Tajima N, et al. Report of the Japan Diabetes Society's committee on the diagnostic criteria for diabetes mellitus and glucose metabolism disorder-a new category of fasting plasma glucose values: "high-normal". J Jpn Diab Soc. 2008;51:281-3.

20. Kawamoto R, Tabara Y, Kohara K, Miki T, Kusunoki T, Takayama S, et al. Low-density lipoprotein cholesterol to highdensity lipoprotein cholesterol ratio is the best surrogate marker for insulin resistance in non-obese Japanese adults. Lipids Health Dis. 2010;9:138.

21. Nakagami T, Tajima N, Oizumi $T$, Karasawa $S$, Wada $K$, Kameda W, et al. Hemoglobin A1c in predicting progression to diabetes. Diabetes Res Clin Pract. 2010;87:126-31.

22. Kasperska-Czyzyk T, Jedynasty K, Bowsher RR, Holloway DL, Stradowska I, Stepień K, et al. Difference in the influence of maternal and paternal NIDDM on pancreatic beta-cell activity and blood lipids in normoglycaemic non-diabetic adult offspring. Diabetologia. 1996;39:831-7.

23. Laakso M, Pyorala K, Voutilainen E, Marniemi J. Plasma insulin and serum lipids and lipoproteins in middle-aged non-insulin dependent diabetic and non-diabetic subjects. Am J Epidemiol. 1987;125:611-21.

24. Lewis GF, Uffelman KD, Szeto LW, Steiner G. Effects of acute hyperinsulinemia on VLDL triglyceride and VLDL apoB production in normal weight and obese individuals. Diabetes. 1993;42: $833-42$.

25. McLaughlin T, Abbasi F, Cheal K, Chu J, Lamendola C, Reaven G. Use of metabolic markers to identify overweight individuals who are insulin resistant. Ann Intern Med. 2003;139:802-9.

26. Oda E, Kawai R, Sukumaran V, Watanabe K. LDL cholesterol is more strongly associated with metabolic syndrome in Japanese women than in men. Intern Med. 2009;48:1607-14.

27. Enomoto M, Adachi H, Hirai Y, Fukami A, Satoh A, Otsuka M, et al. LDL-C/HDL-C ratio predicts carotid intima-media thickness progression better than HDL-C or LDL-C alone. J Lipids. 2011;2011:549137.

28. Takeuchi M, Okamoto K, Takagi T, Ishii H. Ethnic difference in inter-East Asian subjects with normal glucose tolerance and impaired glucose regulation: a systematic review and metaanalysis focusing on fasting serum insulin. Diabetes Res Clin Pract. 2009;82:383-90.

29. Barrett-Connor E, Criqui MH, Witztum JL, Philippi T, Zettner A. Population-based study of glycosylated hemoglobin, lipids, and lipoproteins in nondiabetic adults. Arteriosclerosis. 1987;7:66-70.

30. Khan HA, Sobki SH, Khan SA. Association between glycaemic control and serum lipids profile in type 2 diabetic patients: HbA1c predicts dyslipidaemia. Clin Exp Med. 2007;7:24-9. 